\title{
Seismic Wave Simulatioin Based on Method of Characteristics
}

\author{
Kazuya SHIRAISHI ${ }^{1}$ and Toshifumi MATSUOKA ${ }^{1}$ \\ ${ }^{1}$ Dept. of Civil \& Earth Resources Engineering, Kyoto University, Japan
}

\begin{abstract}
In this paper, we apply the CIP (Cubic Interpolated Profile) method to the simulation of elastic waves as a highly accurate and stable algorithm to solve first-order wave equations. The key idea of the CIP is that not only the physical value itself but also its first spatial derivative obeys the same equations. Using this property the solution is interpolated by cubic polynomials and interpolation coefficients can be evaluated arithmetically. We implemented this idea to the elastic wave simulation by derivation of the first-order wave equations from the basic equations of motion. The derived equations can be interpreted as the combined first-order wave equations for each mode of wave. Then, we define boundary conditions by using the merit of one-side propagation; free surface, solid-fluid boundary, irregular topography. From simulation study and the stability evaluation, we recognize the method of characteristics with the CIP is a very powerful simulation technique for the elastic wave propagation. Numerical dispersion is negligible, requiring about half the number of grid cells per wavelength than other solvers. This allows accurate, high-frequency, full-wavefield simulation in models with highly variable, random elastic contrasts with fluid-solid mixed media and complex topographic media.
\end{abstract}

\section{INTRODUCTION}

In the simulation of elastic waves, the FDM (Finite Difference Method) has been widely adopted because of its simple implementation. However, the numerical dispersion and computational stability are required to be considered carefully. There are some known expanded methods to reduce numerical dispersion, for example; FDM with staggered grids (Vireux, 1986) and the high order differential operator (Levander, 1988).

The computation scheme of the CIP method was proposed by Yabe and Aoki (1991) and had been used in the computational fluid dynamics and plasmas phenomena simulations (Kudoh, 1998; Yabe et al., 2001). We applied this method to the simulation of elastic wave (Shiraishi and Matsuoka, 2005). This method is based on a concept that both the physical property and the spatial derivatives are propagated along the characteristic curves in calculating a hyperbolic differential equation.

In this paper, we describe the derivation of the first-order wave equations for elastic waves, and establish some geophysical boundary conditions; free surface, fluid-solid interface, and irregular topographic boundary. We also show some numerical simulation results in topographic media and randomly inhomogeneous media. We will implement this simulation method to solve the realistic geophysical problems accurately from the macro-scale to the micro-scale.

\section{DERIVATION OF FIRST-ORDER WAVE EQUATION FOR ELASTIC WAVES}

In applying the method of characteristics to multi-dimensional elastic wave modeling, we translate basic formulas in elastic medium into combined first-order wave equation. The equation of continuity and the equation of motion in an elastic medium between stress $\tau$ and particle velocity $v$ are expressed as

$$
\begin{aligned}
\frac{\partial \tau_{i j}}{\partial t} & =\lambda \delta_{i j} \frac{\partial v_{k}}{\partial x_{k}}+\mu\left(\frac{\partial v_{i}}{\partial x_{j}}+\frac{\partial v_{j}}{\partial x_{i}}\right), \\
\frac{\partial v_{i}}{\partial t} & =\frac{1}{\rho} \frac{\partial \tau_{i j}}{\partial x_{j}} .
\end{aligned}
$$

In these equations, $\lambda, \mu$ are Lame's elastic moduli, and $\rho$ is density. We can rewrite these equations in a matrix form with matrices $\mathbf{A}, \mathbf{B}$, and $\mathbf{C}$, which are $9 \times 9$ matrices including Lame's moduli and density, $\frac{\partial}{\partial t}\left[\begin{array}{l}\tau \\ \mathbf{v}\end{array}\right]+\mathbf{A} \frac{\partial}{\partial x_{i}}\left[\begin{array}{l}\tau \\ \mathbf{v}\end{array}\right]+\mathbf{B} \frac{\partial}{\partial x_{j}}\left[\begin{array}{l}\tau \\ \mathbf{v}\end{array}\right]+\mathbf{C} \frac{\partial}{\partial x_{k}}\left[\begin{array}{l}\tau \\ \mathbf{v}\end{array}\right]=\mathbf{0}$

where,

$\left[\begin{array}{ll}\tau & \mathbf{v}\end{array}\right]^{T}=\left[\begin{array}{lllllllll}\tau_{x x} & \tau_{y y} & \tau_{z z} & \tau_{y z} & \tau_{z x} & \tau_{x y} & v_{x} & v_{y} & v_{z}\end{array}\right]^{T}$. 
In translation of formulas, we adopt direction splitting (Takewaki and Yabe, 1987) and diagonalization of matrices in each direction. At first, we separate equation (3) in three directions.Then, we diagonalize the matrices $\mathbf{A}, \mathbf{B}$, and $\mathbf{C}$. Here we show the derivation of the first-order wave equations in $x_{i}$ direction from the equation;

$$
\frac{\partial}{\partial t}\left[\begin{array}{c}
\tau \\
\mathbf{v}
\end{array}\right]+\mathbf{A} \frac{\partial}{\partial x_{i}}\left[\begin{array}{l}
\tau \\
\mathbf{v}
\end{array}\right]=\mathbf{0}
$$

The matrix $\mathbf{A}$ can be transformed with a diagonal matrix $\mathbf{M}$,

$$
\mathbf{A}=\mathbf{L M L}^{-\mathbf{1}} \text {. }
$$

By substituting this relation into equation (4), we can finally an obtain matrix equation,

$$
\frac{\partial}{\partial t} \mathbf{L}^{-1}\left[\begin{array}{l}
\tau \\
\mathbf{v}
\end{array}\right]+\mathbf{M} \frac{\partial}{\partial x_{i}} \mathbf{L}^{-1}\left[\begin{array}{l}
\tau \\
\mathbf{v}
\end{array}\right]=\mathbf{0} .
$$

This matrix formula includes the following nine equations;

$$
\begin{gathered}
\frac{\partial}{\partial t}\left[+\tau_{i i}+I_{p} v_{i}\right]+V_{p} \frac{\partial}{\partial x_{i}}\left[+\tau_{i i}+I_{p} v_{i}\right]=0 \\
\frac{\partial}{\partial t}\left[-\tau_{i i}+I_{p} v_{i}\right]-V_{p} \frac{\partial}{\partial x_{i}}\left[-\tau_{i i}+I_{p} v_{i}\right]=0 \\
\frac{\partial}{\partial t}\left[+\tau_{i j}+I_{s} v_{j}\right]+V_{s} \frac{\partial}{\partial x_{i}}\left[+\tau_{i j}+I_{s} v_{j}\right]=0 \\
\frac{\partial}{\partial t}\left[-\tau_{i j}+I_{s} v_{j}\right]-V_{s} \frac{\partial}{\partial x_{i}}\left[-\tau_{i j}+I_{s} v_{j}\right]=0 \\
\frac{\partial}{\partial t}\left[+\tau_{i k}+I_{s} v_{k}\right]+V_{s} \frac{\partial}{\partial x_{i}}\left[+\tau_{i k}+I_{s} v_{k}\right]=0 \\
\frac{\partial}{\partial t}\left[-\tau_{i k}+I_{s} v_{k}\right]-V_{s} \frac{\partial}{\partial x_{i}}\left[-\tau_{i k}+I_{s} v_{k}\right]=0 \\
\frac{\partial}{\partial t}\left[R_{p s} \tau_{i i}-\tau_{j j}\right]=0 \\
\frac{\partial}{\partial t}\left[R_{p s} \tau_{i i}-\tau_{k k}\right]=0 \\
\frac{\partial \tau_{j k}}{\partial t}=0
\end{gathered}
$$

Here, $I_{p}=\sqrt{\rho(\lambda+2 \mu)}=\rho V_{p}$ and $I_{s}=\sqrt{\rho \mu}=$ $\rho V_{s}$ are $\mathrm{P}$ and $\mathrm{S}$ impedance respectively, and $R_{p s}=$ $\lambda /(\lambda+2 \mu)=1-\mathscr{V}_{s}^{2} / V_{p}^{2}$ is a factor between different normal stresses. $\mathrm{P}$ wave velocity and $\mathrm{S}$ wave velocity are $V_{p}=\sqrt{\lambda+2 \mu / \rho}$ and $V_{s}=\sqrt{\mu / \rho}$.

Equations (7) and (8) show $\mathrm{P}$ wave propagation in forward direction and backward direction respectively, and equations (9) to (12) represent $S$ wave propagations in each directions of two different modes. We can interpret equation (9) and (10) describe SV wave propagation, and equation (11) and (12) describe SH wave propagation. Equations (13) to (15) show relationships between stress components of different directions. In 3D case, we have totally $9 \times 3=27$ equations.

\section{CIP METHOD}

Wave propagation in an alternative way is expressed by an equation

$$
\frac{\partial f}{\partial t}+u \frac{\partial f}{\partial x}=0 .
$$

This first-order wave equation means that wave packet $f$ propagate along the curve $d x / d t=u$. This curve is called a characteristic curve and equation (16) is interpreted as a first-order wave equation for forward wave propagation. Although this equation has a simple form, it had been difficult to calculate numerically with high stability and less numerical dispersion. The CIP method overcomes this problem by solving the equation with a spatial derivative.

If we assume that propagation velocity $u$ is constant, we obtain a same equation for $g$ which is the spatial derivative of $f$ by differentiating equation (16) with spatial variable $x$,

$$
\frac{\partial g}{\partial t}+u \frac{\partial g}{\partial x}=0, \quad g=\frac{\partial f}{\partial x} .
$$

These equations 16 and 17 show that both the physical property and the spatial derivatives are propagate along the same characteristic curves. The CIP method is based on this concept in calculating a hyperbolic differential equation. Fig.1 shows conceptual diagrams of the CIP method. Solid line is initial profile and dashed line is an exact solution after propagation (Fig.1(a)). When the solutions after one time step propagation are interpolated linearly, numerical diffusion appears (Fig.1(b)). In the CIP method, the spatial derivatives also propagate and the profile inside a grid cell is retrieved (Fig.1(c)).

If two values of physical property $f$ and the spatial derivative $g$ are given at two grid points, $x_{i}$ and $x_{i+1}$, the profile between these points can be interpolated by a cubic polynomial,

$$
F_{i}(x)=a_{i}\left(x-x_{i}\right)^{3}+b_{i}\left(x-x_{i}\right)^{2}+c_{i}\left(x-x_{i}\right)+d_{i} .
$$

In addition, there are the following four constraints 
on $f$ and $g$ at the grid points

$$
\begin{aligned}
& F_{i}\left(x_{i}\right)=f_{i}=d_{i}, \\
& \frac{d F_{i}\left(x_{i}\right)}{d x}=g_{i}=c_{i}, \\
& F_{i}\left(x_{i-1}\right)=f_{i-1} \\
& \quad=-a_{i} \Delta x^{3}+b_{i} \Delta x^{2}-c_{i} \Delta x+d_{i}, \\
& \frac{d F_{i}\left(x_{i-1}\right)}{d x}=g_{i-1} \\
& \quad=3 a_{i} \Delta x^{2}-2 b_{i} \Delta x+c_{i}, \\
& \Delta x=x_{i}-x_{i-1} .
\end{aligned}
$$

From above equations, the profiles of $f$ and $g$ at the $n+1$ th step are obtained by shifting the previous profiles by $u_{i} \Delta t$,

$$
\begin{aligned}
& f_{i}^{n+1}=a_{i} \xi^{3}+b_{i} \xi^{2}+g_{i}^{n} \xi+f_{i}^{n}, \\
& g_{i}^{n+1}=3 a_{i} \xi^{2}+2 b_{i} \xi+g_{i}^{n}, \\
& \xi=-u_{i} \Delta t .
\end{aligned}
$$

The coefficients in equations (23) and (24), $a_{i}$ and $b_{i}$, are obtained by evaluating the above constraints,

$$
\begin{aligned}
& a_{i}=\frac{g_{i}^{n}+g_{i u p}^{n}}{D^{2}}+\frac{2\left(f_{i}^{n}-f_{i u p}^{n}\right)}{D^{3}}, \\
& b_{i}=\frac{3\left(f_{i} u p^{n}-f_{i}^{n}\right)}{D^{2}}-\frac{2 g_{i}^{n}+g_{i u p}^{n}}{D} .
\end{aligned}
$$

Here, $D=-\Delta x$, iup $=i-1$ for $u \geq 0$ (forward propagation) and $D=\Delta x$, iup $=i+1$ for $u<0$ (backward propagation).

We analyze stability and dispersion features of the CIP scheme by von Neumann's method. Fig.2 shows the gain and phase of amplification factor $\mathrm{G}(\mathrm{k})$ obtained by substituting $f_{i}^{n}=G \exp (-j k j \Delta x)$ $(j=\sqrt{-1})$ to the first-order wave equation (equation (16)) about five difference schemes; CIP scheme, first order up-wind scheme (UPW), Lax-Wendroff scheme (LXW), second order central difference scheme (FDC), and Crank-Nicholson scheme (C-N).

\section{ELASTIC WAVE SIMULATION BY CIP METHOD}

In the modeling of elastic waves based on the equations (7) to (12) are calculated by the CIP scheme and equations (13) to (14) are calculated by the central difference approximation. In the CIP method, we need to evaluate the equations for the spatial derivatives, too. (a)

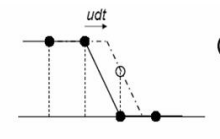

(b)

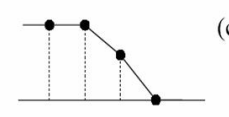

(c)

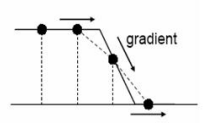

Figure 1. Conceptual diagrams of the CIP method.
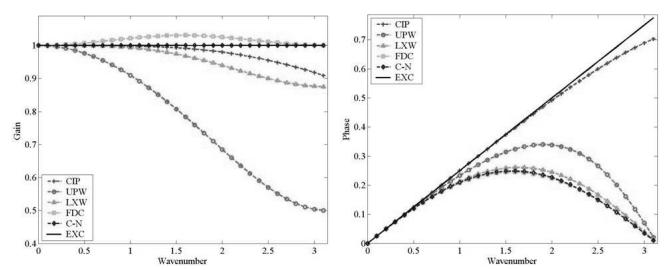

Figure 2. The gain (left) and the phase curves (right) at the first step with analytical spatial derivatives at $u \Delta t / \Delta x=0.25$. CIP scheme (CIP), up-wind scheme (UPW), Lax-Wendroff scheme (LXW), central difference scheme (FDC), Crank-Nicholson scheme (C-N), and exact solution(EXC).

We show an example of calculation of the equations (7) and (8). The forward propagation of $+\tau_{i i}+I_{p} v_{i}$ and the backward propagation of $-\tau_{i i}+$ $I_{p} v_{i}$ are calculated from the equation (7) and (8) respectively;

$$
\begin{aligned}
& {\left[+\tau_{i i}^{n}+I_{p} \cdot v_{i}^{n}\right]} \\
& {\left[-\tau_{i i}^{n}+I_{p} \cdot v_{i}^{n}\right]}
\end{aligned} \quad \longrightarrow \quad\left[+\tau_{i i}^{+}+I_{p} \cdot v_{i}^{+}\right]
$$

Here superscripts ${ }^{+}$and ${ }^{-}$mean the forward and the backward propagation. Then we calculate the wavefield at the $n+1$ th step by linear summation of them;

$$
\begin{aligned}
\tau_{i i}^{n+1} & =\frac{1}{2}\left[\left(\tau_{i i}^{+}+\tau_{i i}^{-}\right)+\left(v_{i}^{+}-v_{i}^{-}\right) \cdot I_{p}\right], \\
v_{i}^{n+1} & =\frac{1}{2}\left[\left(v_{i}^{+}+v_{i}^{-}\right)+\left(\tau_{i i}^{+}-\tau_{i i}^{-}\right) / I_{p}\right] .
\end{aligned}
$$

The stress $\tau_{i i}$ and the particle velocity $v_{i}$ are related by the impedance $I_{p}$. In actual calculation, we calculate both forward and backward propagation for each variable. In addition, their spatial derivatives are also calculated by the same relationship as above equations,

$$
\begin{aligned}
& \partial_{i} \tau_{i i}^{n+1}=\frac{1}{2}\left[\left(\partial_{i} \tau_{i i}^{+}+\partial_{i} \tau_{i i}^{-}\right)+\left(\partial_{i} v_{i}^{+}-\partial_{i} v_{i}^{-}\right) \cdot I_{p}\right], \\
& \partial_{i} v_{i}^{n+1}=\frac{1}{2}\left[\left(\partial_{i} v_{i}^{+}+\partial_{x} v_{i}^{-}\right)+\left(\partial_{i} \tau_{i i}^{+}-\partial_{i} \tau_{i i}^{-}\right) / I_{p}\right] .
\end{aligned}
$$

Here, we defined $\partial_{i}$ as a spatial derivative $\partial / \partial x_{i}$.

The total wavefield in a multi-dimensional media is calculated by the equations in all directions in 


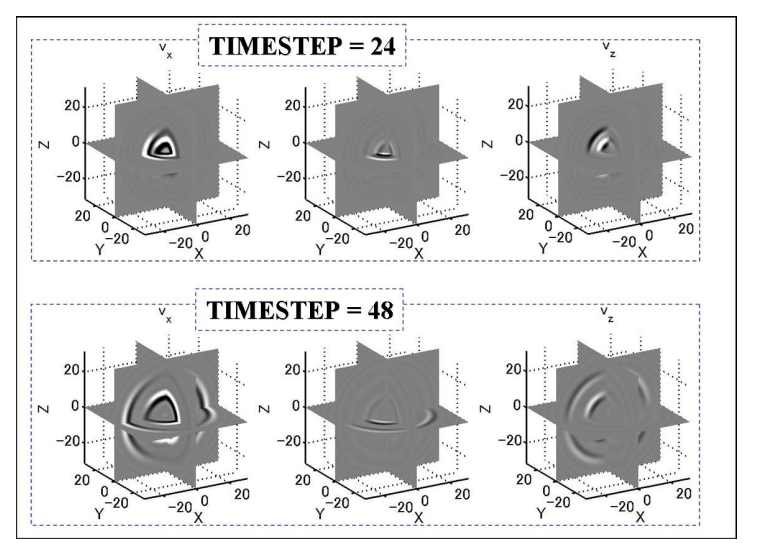

Figure 3. Three dimensional elastic wave propagation.

order based on the concept on the direction splitting. Fig.3 shows an numerical example of elastic wave in a three dimensional homogeneous media when the particle velocity in $x$ direction is applied as a source at the center. As shown in the snapshots, $\mathrm{P}$ wave and $\mathrm{S}$ wave propagate appropriately.

\section{BOUNDARY CONDITIONS}

For the elastic wave simulation in geophysics, we need to set some boundary conditions; free surface (e.g. earth surface), fluid-solid interface (e.g. sea floor), transparent / absorbing boundary (e.g. semi-infinite medium), etc. The physical boundary conditions are described by physical values themselves $\left\{\tau_{i j}, v_{i}\right\}$ and their spatial derivatives $\left\{\partial \tau_{i j} / \partial x_{k}, \partial v_{i} / \partial x_{j}\right\}$. In the wave simulation based on the method of characteristics with the CIP method, we can satisfy the conditions theoretically, since we calculate the wave propagation based on the feature of solving first-order equation and calculation both physical values and their spatial derivatives. In calculation at the $n+1$ th time step wavefield by equations (30) to equation (33) at the boundaries, we consider the sign of the propagated wavefield and spatial derivatives.

Here we show four kinds of boundary conditions; free surface, fluid-solid interface, and transparent / absorbing boundary. To simplify the problems, we treat two dimensional case and we assume the boundary is located at $z=j \Delta z$.

\section{(1) Free surface}

The free surface is the most important boundary

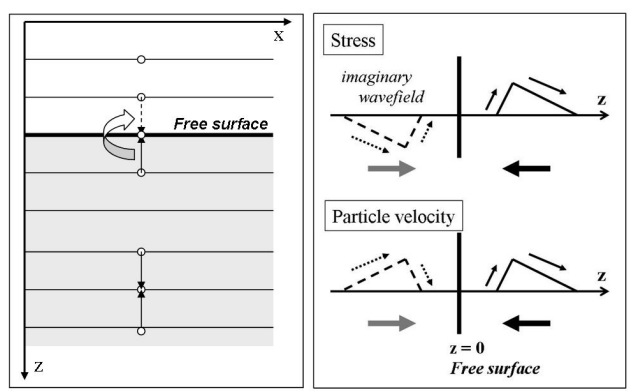

Figure 4. Free surface boundary condition and imaginary wave field.

for elastic waves. The conditions are expressed as

$$
\begin{gathered}
\tau_{z z}=0, \quad \tau_{z x}=0 \\
\frac{\partial v_{x}}{\partial z}=0, \quad \frac{\partial v_{z}}{\partial z}=0 .
\end{gathered}
$$

We calculate stress and particle velocity at the free surface using underground wave and mirrored imaginary wave above the surface with opposite or same sign respectively (see Fig.4). It is also important to consider the sign of both the physical values and the spatial derivatives. From the calculation process at the $n+1$ th wave field represented in equations (30) to (33), we need to prepare the forward and backward propagated wavefield. To satisfy the conditions in equations (34) and (35), we set the forward propagated wavefield using backward propagated wavefield as follows; for the stress,

$$
\begin{array}{cl}
\tau_{z z}^{+}=-\tau_{z z}^{-}, & \tau_{z x}^{+}=-\tau_{z x}^{-}, \\
\partial_{z} \tau_{z z}^{+}=\partial_{z} \tau_{z z}^{-}, & \partial_{z} \tau_{z x}^{+}=\partial_{z} \tau_{z x}^{-},
\end{array}
$$

and for the particle velocity,

$$
\begin{gathered}
v_{x}^{+}=v_{x}^{-}, \quad v_{z}^{+}=v_{z}^{-}, \\
\partial_{z} v_{x}^{+}=-\partial_{z} v_{x}^{-}, \quad \partial_{z} v_{z}^{+}=-\partial_{z} v_{z}^{-} .
\end{gathered}
$$

\section{(2) Fluid-solid interface}

The fluid-solid interface is also a main boundary for the elastic wave. The sea bottom is a representative boundary in the local seismology and the seismic exploration, and the interface between pore fluid and rock matrix also important in micro-scale geophysics. At the fluid-solid interface, we need to consider continuities about normal stress and particle velocity in $z$ direction,

$$
\tau_{z x}=0, \quad \frac{\partial \tau_{z z}}{\partial z}=0, \quad \frac{\partial v_{z}}{\partial z}=0 .
$$




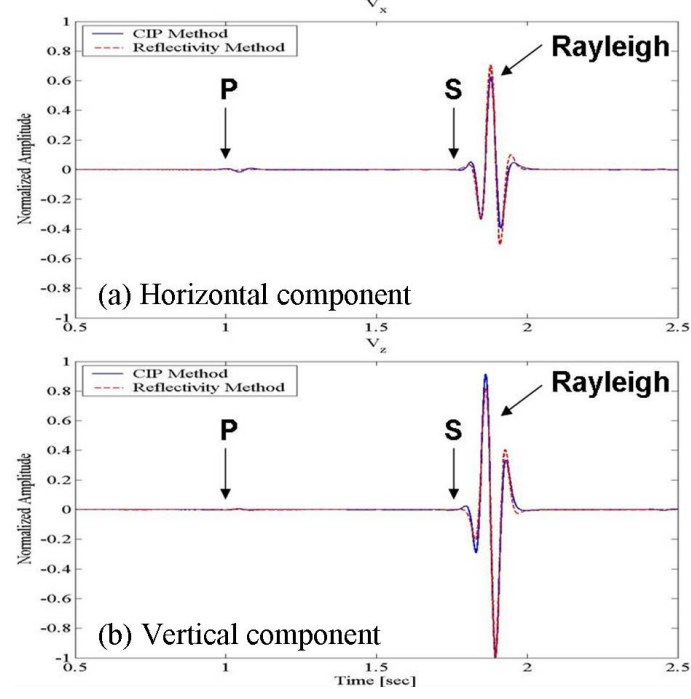

Figure 5. Solutions of Lamb's problem by CIP method (solid line) and reflectivity method (dashed line). (a) is horizontal component, and (b) is vertical component at $x=4,000 \mathrm{~m}$.

To satisfy these conditions, we applied a same technique for settings of forward and backward wavefields as shown above for the free surface.

\section{(3) Transparent / absorbing boundary}

The CIP method naturally satisfies transparent boundary condition in an orthogonal direction to the boundary at each edge, because it basically solves first-order wave equations throughout. However, oblique incident waves at edges are not disappear perfectly. Especially, S wave with high incident angle reflect/refract at the edge. We recommend to combine the absorbing boundary by amplitude damping (e.g. Cerjan, 1985) with the transparent boundary based on the first-order wave propagation.

\section{NUMERICAL EXAMPLES}

\section{(1) Lamb's problem}

In order to evaluate the boundary condition for the free surface, we attempt to solve Lamb's problem. We applied a vertical point force $(10 \mathrm{~Hz}$ Ricker wavelet) on a homogeneous half-space $\left(V_{p}=\right.$ $\left.4,000 \mathrm{~m} / \mathrm{s}, V_{s}=2,309 \mathrm{~m} / \mathrm{s}, \rho=2,500 \mathrm{~kg} / \mathrm{m}^{3}\right)$. Grid spacing is $\Delta x=\Delta z=10 \mathrm{~m}$ with $N X \times N Z=$ $501 \times 251$ grids, and time interval for calculation is $\Delta t=0.001 \mathrm{~s}$. Fig.5 shows seismograms at $x=4,000 \mathrm{~m}$ and comparison with the result from the reflectivity method. We can appropriately calculate the wave propagation in the near surface including Rayleigh wave.
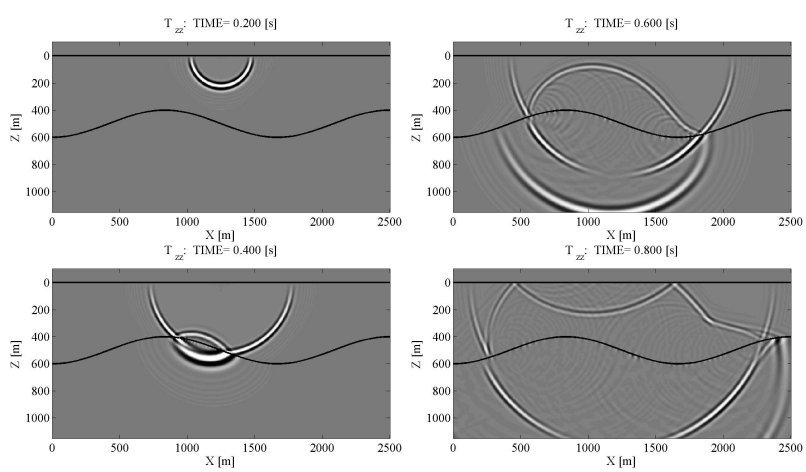

Figure 6. Snapshots of $\tau_{z z}$ component propagating in a fluid-solid interface model.

\section{(2) Irregular topographic media}

The second model has the sinusoidal wavy variation at the seafloor.We assumed the upper layer as sea, $V_{p}=1,500 \mathrm{~m} / \mathrm{s}, V_{s}=0 \mathrm{~m} / \mathrm{s}, \rho=1,030$ $\mathrm{kg} / \mathrm{m}^{3}$, and the lower layer as Poisson solid, $V_{p}=$ $3,000 \mathrm{~m} / \mathrm{s}, V_{s}=1,732 \mathrm{~m} / \mathrm{s}, \rho=2,500 \mathrm{~kg} / \mathrm{m}^{3}$. Grid spacing is $\Delta x=\Delta z=5 \mathrm{~m}$ with $N X \times N Z=$ $501 \times 251$ grids, and time interval is $\Delta t=0.001 \mathrm{~s}$. We applied pressure source ( $30 \mathrm{~Hz}$ Ricker wavelet) at $x=1,250 \mathrm{~m}$ and $z=10 \mathrm{~m}$. Fig.6 shows a snapshots of $\tau_{z z}$ component (pressure in the sea). The incident $P$ wave reflected, and transmitted wave and converted $S$ wave are occurred at the seafloor.

Our boundary conditions work appropriately not only at flat boundaries but also curved boundaries in both the free surface and the fluid-solid interface. We realized the boundary conditions to deal with realistic geophysical problems.

\section{(3) Random media}

The final example deal with the randomly inhomogeneous medium. The $\mathrm{P}$ wave model shown in Fig.7 is generated by random number and moving average, and $\mathrm{S}$ wave velocity is defined by $V_{p} / V_{s}=$ $\sqrt{3}$ and density $\rho=2.5$ is constant. The model has relatively high velocity contrasts. Grid spacing is $\Delta x=\Delta z=10 \mathrm{~m}$ with $N X \times N Z=256 \times 256$ grids, and time interval is $\Delta t=0.001 \mathrm{~s}$. The particle velocity (vertical, $30 \mathrm{~Hz}$ Ricker wavelet) is applied at the center of the model as a source. Fig.8 shows snapshots of $\mathrm{P}$ and $\mathrm{S}$ wave propagation at $t=0.4 \mathrm{~s}$ by the CIP method and by the fourth order finite difference method with staggered grid (FDSG). Although both snapshots represent similar scattering wavefields in the random medium, the FDSG shows numerical dispersion after $\mathrm{S}$ wave propagation. This is caused by the shorter wave length of $\mathrm{S}$ wave than 

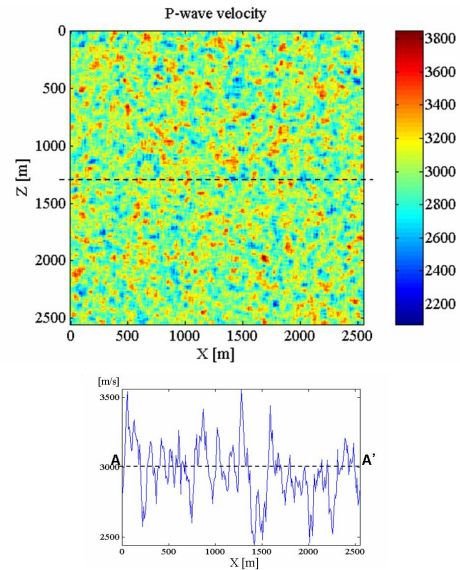

Figure 7. Distribution of P wave velocity; (left) $x-y$ plot, and (right) cross section at line AA'.

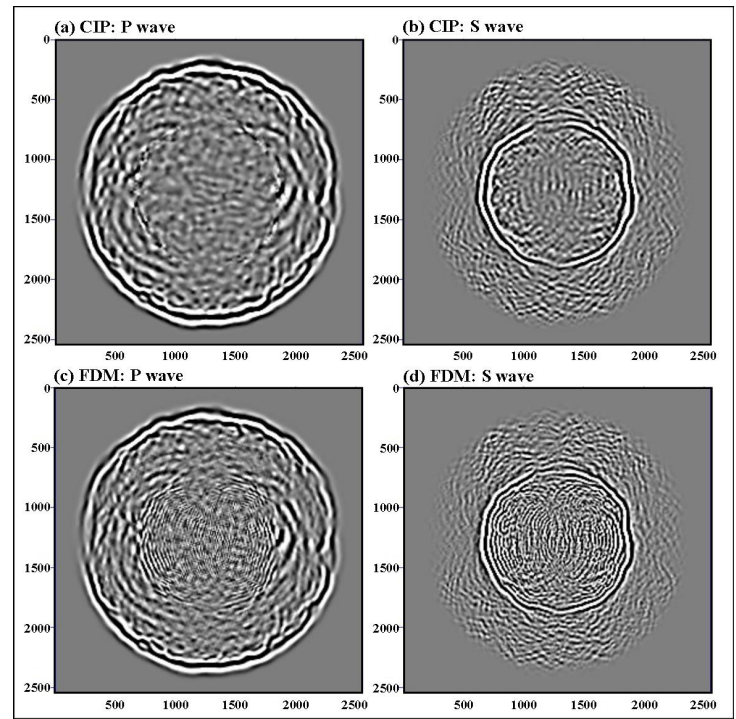

Figure 8. Snapshots $\mathrm{P}$ wave and $\mathrm{S}$ wave component calculated by divergence and curl at $t=0.4 \mathrm{~s}$. (a) and (b) the CIP method, (c) and (d) Finite-Difference Method with Staggered-Grids.

that of $\mathrm{P}$ wave. These results shows that the CIP method can simulate the wave propagation with less numerical dispersion.

\section{CONCLUSIONS}

We applied the CIP scheme to the wave modeling based on the method of characteristics. The first-order wave equations for the elastic waves are derived from the basic equations of motion. For the realistic wave modeling, we established some geophysical boundary conditions for this method. Since the CIP method calculate the propagations of both physical values and their spatial derivatives, we can simulate elastic wave propagation with stability and less numerical dispersion and we can satisfy the boundary conditions theoretically. We conclude the CIP scheme is a very powerful technique to deal with geophysical problems such as accurate, high-frequency, full-wavefield simulation in models with highly variable, random elastic contrasts with fluid-solid mixed media and complex topographic media.

\section{REFERENCES}

1) Cerjan, C., Kosloff, D., Kosloff, R., and Reshef, M. (1985): A nonreflecting boundary condition for discrete acoustic and elastic wave equations, Geophysics, 50, 705-708.

2) Kudoh, T. (1998): Magnetically driven jets from accretion disks. III. 2.5-dimensional nonsteady simulations for thick disk case, The Astrophysical Journal, 508, 186-199.

3) Levander, A. R. (1988): Fourth-order finite-difference P-SV seismograms, Geophysics, 53, 1425-1436.

4) Shiraishi, K. and Matsuoka, T. (2005): Elastic modeling based on advection equations, 67th EAGE Conference \& Exhibition, Extended Abstracts, P264.

5) Takewaki, H., and Yabe, T. (1987): The Cubic- interpolated Pseudo Particle (CIP) Method: Application to Nonlinear and Multi-dimensional Hyperbolic Equations, J. Comput. Phys., 70, 353-372.

6) Vireux, J. (1986): P-SV wave propagation in heterogeneous media: Velocity-stress finite- difference method, Geophysics, 51, 889-901.

7) Yabe, T. and Aoki, T. (1991): A universal solver for hyperbolic equation by cubic-polynomial interpolation, I. One-dimensional solver, Comput. Phys. Commun., 66, 219-232.

8) Yabe, T., Xiao, F., and Utsumi, T. (2001): The Constrained Interpolation Profile Method for Multiphase Analysis, $J$. Comput. Phys., 169, 556-593. 